\title{
Clinical and biological significance of adamantinomatous craniopharyngioma with CTNNB1 mutation
}

\author{
Takuma Hara, MD, ${ }^{1}$ Hiroyoshi Akutsu, MD, PhD, ${ }^{1}$ Shingo Takano, MD, PhD, ${ }^{1}$ Hiroyoshi Kino, MD, ${ }^{1}$ \\ Eiichi Ishikawa, MD, PhD, ${ }^{1}$ Shuho Tanaka, MD, PhD, ${ }^{2}$ Hidetaka Miyamoto, MD, ${ }^{2}$ \\ Noriaki Sakamoto, MD, PhD, ${ }^{3}$ Keiichiro Hattori, MD, ${ }^{4}$ Mamiko Sakata-Yanagimoto, MD, PhD, ${ }^{4}$ \\ Shigeru Chiba, MD, PhD, ${ }^{4}$ Takashi Hiyama, MD, ${ }^{5}$ Tomohiko Masumoto, MD, PhD, ${ }^{5}$ and \\ Akira Matsumura, MD, $\mathrm{PhD}^{1}$
}

Departments of ${ }^{1}$ Neurosurgery, ${ }^{2}$ Otolaryngology, ${ }^{3}$ Diagnostic Pathology, ${ }^{4}$ Hematology, and ${ }^{5}$ Radiology, Faculty of Medicine, University of Tsukuba, Tsukuba, Ibaraki, Japan

OBJECTIVE The Wnt/ $\beta$-catenin signaling pathway is strongly implicated in the pathogenesis of adamantinomatous craniopharyngioma (adaCP). However, there is no evidence that the CTNNB1 mutation activates the target gene of Wnt/ $\beta$ catenin signaling, and it is unknown whether it affects the tumorigenesis of adaCP. To assess the effect of the CTNNB1 mutation of adaCP, the authors analyzed the correlation between the mutation and clinical, radiological, pathological, and biological findings.

METHODS Between 2003 and 2015, 42 patients (24 male and 18 female, median age 42 years) with either papillary craniopharyngioma (papCP) or adaCP underwent tumor resection at the authors' institution. BRAF V600E and CTNNB1 in papCP and adaCP samples were sequenced by next-generation sequencing and the Sanger method, and mRNA expression levels of Axin2 and BMP4 were evaluated by RT-PCR. Axin2, BMP4, $\beta$-catenin, and BRAF expression were evaluated by immunohistochemistry. Other data were collected from clinical reports.

RESULTS The BRAF V600E mutation was detected in all 10 cases of papCP (100\%). CTNNB1 exon 3 mutations were detected in 21 of $31(68 \%)$ cases of adaCP, excluding 1 case for which there were no available sequence data. The mRNA expression level of Axin2 was significantly higher in adaCPs with a CTNNB1 mutation than in those without ( $p<$ 0.05). The immunohistochemical findings of Axin2 and BMP4 did not correlate with CTNNB1 mutation positivity. When patients who received adjuvant radiation therapy were excluded, progression-free survival was shorter in the mutationpositive group than in the mutation-negative group (log-rank test, $p=0.031$ ). Examination of clinical characteristics and immunohistochemical findings of adaCPs showed that there was no significant correlation between CTNNB1 mutation positivity and age, sex, tumor volume, gross-total resection, optic tract edema, calcification, or T1 signal intensity of cyst fluid on MRI, $\beta$-catenin, and MIB-1 index.

CONCLUSIONS These results raise the possibility that the CTNNB1 mutation in adaCP may be associated with disease recurrence, and genes related to the Wnt/ $\beta$-catenin signaling pathway might represent a therapeutic target.

https://thejns.org/doi/abs/10.3171/2018.3.JNS172528

KEYWORDS BRAF; craniopharyngioma; CTNNB1; Wnt/ק-catenin; oncology

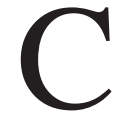
RANIOPHARYNGIOMAS (CPs) are histologically benign, solid or mixed solid-cystic epithelial tumors of the sellar and suprasellar region. ${ }^{8}$ They originate from the epithelium of Rathke's pouch and are divided into adamantinomatous-type $\mathrm{CP}(\mathrm{adaCP})$ and papillarytype CP (papCP) according to the World Health Organization classification. ${ }^{18}$ These 2 subtypes differ not only in histopathology but also in epidemiological, radiological, genomic, and transcriptomic characteristics. ${ }^{26} \mathrm{CPs}$ are the third most common pediatric brain tumors and account for $2.3 \%$ of all primary intracranial neoplasms. ${ }^{5}$ AdaCP is the most common non-neuroepithelial intracerebral neoplasm in children. ${ }^{12,27}$ Treatment of both types of CPs remains difficult due to anatomical proximity of these le-

ABBREVIATIONS ada = adamantinomatous; $C P=$ craniopharyngioma; FFPE = formalin-fixed paraffin-embedded; GSK3- $\beta=$ glycogen synthase kinase 3- $\beta$; GTR = grosstotal resection; IHC = immunohistochemistry; NGS = next-generation sequencing; pap = papillary; $P F S=$ progression-free survival; $R T$ = radiation therapy; $R$ T-PCR = realtime polymerase chain reaction.

SUBMITTED October 10, 2017. ACCEPTED March 1, 2018.

INCLUDE WHEN CITING Published online August 3, 2018; DOI: 10.3171/2018.3.JNS172528. 
sions to important functional structures, for example, the optic nerve, hypothalamus, and pituitary stalk. Achieving gross-total resection (GTR) of either type of CP without any complications remains challenging, ${ }^{5}$ and non-GTR results in recurrence requiring reoperation or adjuvant radiation therapy (RT). In Japan, the 5-year overall survival rate is $96.5 \%$ for patients with either type of $\mathrm{CP}$, and the 5 -year progression-free survival (PFS) rate is $69.7 \% ;{ }^{5}$ recurrence of tumor in young patients is problematic. The long-term management of both types of CPs is difficult and complex, and there is no clear consensus on the optimal management of residual tumor.

In recent years, the $W n t / \beta$-catenin signaling pathway has been strongly implicated in the pathogenesis of adaCP. Genetic analyses have shown that $64 \%-99 \%$ of adaCPs have activating mutations in exon 3 of the $\beta$-catenin gene CTNNB1. ${ }^{1,3,4,12,26}$ CTNNB1 mutation causes aberrant $\beta$ catenin protein accumulation and translocation to the nucleus, where it facilitates transcription of $\beta$-catenin target genes and stimulates cellular proliferation and other Wnt-regulated cellular processes. ${ }^{2-4,16}$ However, there is no clear evidence that $C T N N B 1$ mutation activates the target genes of Wnt signaling (AXIN2, BMP4, etc.), and it is unknown whether it affects the tumorigenesis of adaCPs.

In previous reports, certain factors, including tumor volume, histopathologic type, lesion position, degree of residual tumor after resection, region of invasion, and aberrant membranous expression of $\beta$-catenin, have been shown to correlate with the risk of recurrence. ${ }^{17,21}$ However, the relationship between the biological findings of both types of CPs and PFS remains unclear. Furthermore, the clinical, radiological, pathological, and biological features of adaCP with CTNNBI mutation and the features of adaCP without $C T N N B 1$ mutation are unclear. From the standpoint of treatment, although the BRAF V600E mutation has been identified as a molecular and therapeutic target of papCP, ${ }^{14,15}$ the therapeutic target of adaCP remains unknown.

In this study, in order to assess the effect of the CTNNBI mutation in adaCP, we examined whether a positive mutation in adaCP is present using targeted next-generation and Sanger sequencing. Using those data, we analyzed the correlation between the mutation and clinical, radiological, pathological, and biological findings (Wnt/ $\beta$-catenin signaling pathway).

\section{Methods \\ Patients}

This study was approved by the ethics committee of University of Tsukuba Hospital, and informed consent was obtained from the patients or their parent/guardian. Thirtytwo patients with adaCP and 10 patients with papCP as the control group - patients whose cases had been diagnosed at the department of pathology in our university-were included in this study. The operations were performed by senior surgeons (S. Tanaka and H.A.) at our hospital from 2003 to 2015. All patients underwent endoscopic endonasal or transcranial surgery depending on tumor localization. However, we performed endoscopic transventricular cyst fenestration or intended partial/subtotal tumor resec- tion followed by adjuvant RT in some adults, especially elderly patients in whom aggressive resection might result in poor performance status or severely impaired quality of life. If postoperative adjuvant RT was needed, fractionated radiotherapy or CyberKnife treatment was initiated within 3 months after surgery. In treating pediatric patients, we tried to avoid RT in order to prevent late radiation toxicity. The following were inclusion criteria for this study: 1) availability of hematoxylin and eosin-stained sections for histological review of the case; and 2) availability of formalin-fixed paraffin-embedded (FFPE) tissue for molecular characterization (extraction of DNA and RNA). A total of 62 surgeries for $\mathrm{CP}$ were performed in 54 patients during the study period. Twelve patients who did not meet the inclusion criteria were excluded, because we could not obtain sufficient tissue sample for extracting DNA and RNA in some cases of adaCP, especially in cases in which endoscopic transventricular cyst fenestration was performed, followed by RT. As a result, 42 patients were included in this study. Histologically, 10 (24\%) cases were papCPs and 32 (76\%) were adaCPs. PFS was analyzed in adaCP patients with at least 12 months of follow-up. PFS time was defined as the interval between surgery and confirmation of recurrence by radiological findings.

\section{Histology and Immunohistochemistry}

All tissue specimens were fixed in $10 \%$ formaldehyde and paraffin embedded according to routine protocols. Serial $4-\mu m$-thick sections were then prepared, and immunostaining for Ki67 (Immunotech Laboratories Inc.) was performed. Ki67 positivity of nuclei was determined by counting at least 1000 tumor cells in a homogeneously stained area. Immunostaining was performed with a commercially available polyclonal Axin2 antibody (ab32197, dilution 1:500; Abcam plc) at $1 \mathrm{mg} / \mathrm{ml}$; a polyclonal $B M P 4$ antibody (ab39973, dilution 1:100; Abcam) at $1 \mathrm{mg} / \mathrm{ml}$; a $B R A F$ V600E antibody (E19290, dilution 1:50; Spring Bioscience); and an LSAB2 kit (DAKO) with Tris-EDTA antigen retrieval buffer ( $\mathrm{pH}$ 9.0). Immunostaining was also conducted with a monoclonal $\beta$-catenin antibody (M3539, dilution 1:200; DAKO) at $58.7 \mu \mathrm{g} / \mathrm{ml}$ and EnVision + Dual Link (DAKO).

All histological findings were independently evaluated by 2 different observers (N.S. and H.K.). The observers were blinded to patients' data. When discrepancies occurred, the 2 observers discussed the case to reach a consensus. Cases with $>50 \%$ of cells stained were defined as strongly positive (++; Axin $2, B M P 4, \beta$-catenin), and cases with $<50 \%$ of cells stained were defined as weakly positive $(>10 \%,+)$ or negative $(<10 \%,-)$. "Catenin positive" means positive catenin nuclear staining. In this study, strong and weak positivity on immunohistochemistry (IHC) were regarded as positive findings. Negative controls (X090302, DAKO) at the same protein concentration to primary antibody were used for evaluation of IHC (Axin2, BMP4).

\section{DNA and cDNA Preparation}

For DNA extraction, snap-frozen $\left(-80^{\circ} \mathrm{C}\right)$ and FFPE tissue samples were used. The frozen sections of all tissue 
samples were microscopically analyzed to confirm tumor content. We collected DNA from FFPE and frozen tissue as much as possible. DNA in frozen and FFPE tissue was extracted using DNeasy Blood \& Tissue Kit (Qiagen) and QIAamp DNA Mini Kit (Qiagen), respectively.

For RNA extraction, the FFPE sample was scraped under microscope guidance from three 10- $\mu$ m-thick sections, using RNEasy FFPE kit (Qiagen). RNA of adaCP was considered to be of sufficient purity at A260/A230 and A260/A280 ratios of RNA > 1.7 measured by Nano Drop 2000 (Thermo Fisher Scientific). The median RNA integrity number was $2.4(1.8-2.5)$ measured by Agilent 2100 bioanalyzer (Agilent Technologies) and 31 of 32 RNA integrity numbers fell within the range of 2.3-2.5. Subsequently, for reverse transcription, SuperScript IV Master Mix with ezDNase Enzyme (Thermo Fisher Scientific) was used according to the manufacturer's instructions.

\section{Mutation Analysis}

Genomic DNA was used for each amplification reaction using primers specific for $B R A F$ and for CTNNBI. We prepared 2 different primers: one for next-generation sequencing (NGS) and one for Sanger sequencing. The polymerase chain reaction (PCR) was performed according to the manufacturer's instructions for KOD-plus-neo (TOYOBO). The NGS primer sequence of $B R A F$ forward was 5'-TTTCCTTTACTTACTACACCTCAGA-3' and the reverse was 5'-CCATCCACAAAATGGATCCAGAC-3'. The primer sequence of $C T N N B 1$ forward was $5^{\prime}$-ATGG CCATGGAACCAGACAG-3' and the reverse was $5^{\prime}$-TG GGAGGTATCCACATCCTCT-3'. NGS targeted sequencing was performed for all cases. The libraries were made by using the Ion Plus fragment library kit (Life Technologies) according to the protocol described in "Prepare Amplicon Libraries Requiring Fragmentation Using the Ion Xpress Plus Fragment Library Kit" (available at thermofisher.com). PCR amplicons were ligated to barcoded adapters and P1 adapters and then amplified. The libraries were then subjected to deep sequencing on Ion Torrent PGM according to the standard protocol using Ion 318 chips (Thermo Fisher Scientific). The data were analyzed by Valiant caller 4.2 (Thermo Fisher Scientific). Singlenucleotide variants or insertions/deletions with a frequency $\geq 1 \%$ were adopted as mutations. ${ }^{19}$ All candidate mutations were validated by genomic PCR followed by Sanger sequencing. The Sanger primer sequence of $B R A F$ forward was 5'-TGCTTGCTCTGATAGGAAAATG-3' and the reverse was 5'-CCACAAAATGGATCCAGACA-3'. The primer sequence of $C T N N B 1$ forward was $5^{\prime}$-GGCC ATGGAACCAGACAGAA-3' and the reverse was 5'-GC ATTCTGACTTTCAGTAAGGCAAT-3'.

\section{mRNA Expression Level by RT-PCR}

Axin2 is a well-recognized inhibitor and target gene of $\beta$-catenin. ${ }^{14}$ BMP4 expression is said to be enhanced in tumors with oncogenic $\beta$-catenin elevation. ${ }^{15}$ Axin2 and BMP4 are well-known indicators of Wnt signaling activated by nuclear $\beta$-catenin accumulation in cells. Relative mRNA expression was analyzed by real-time poly- merase chain reaction (RT-PCR) using specific TaqMan assays (Applied Biosystems): Axin2 (Hs01063170_m1) and BMP4 (Hs00370078_m1). The values were normalized with respect to the mean expression of the endogenous control: PGK1 (Hs99999906_m1). The reactions were performed according to the manufacturer's recommendation, and relative mRNA expression values were determined using the $2^{-\Delta \Delta \mathrm{Ct}}$ method.

\section{Evaluation of Radiological Findings}

All radiological findings of adaCPs and papCPs were independently evaluated by 2 neuroradiologists (T.M. and T.H.), who were blinded to patient data. When discrepancies occurred, the 2 neuroradiologists discussed the case to reach a consensus. We evaluated tumor volume by MRI (estimated by [AP $\times \mathrm{LR} \times \mathrm{SI}] / 2$, where AP is the anteroposterior measurement, LR is the left-right measurement, and SI is the superior-inferior measurement). Edema of the optic tract,,$^{23}$ calcification, and high intensity of cyst fluid on T1-weighted MRI are the representative radiological findings of adaCPs. ${ }^{8,16}$ All of these findings were evaluated on preoperative MRI and CT to investigate whether there was a correlation between the mutation and any radiological finding.

\section{Statistical Analyses}

All statistical analysis was performed using SPSS 22.0 software (IBM Corp.). The chi-square test or Fisher's exact test was used to evaluate relationships between 2 variables. Differences between variables were evaluated using the Mann-Whitney U-test. PFS analysis was performed using the Kaplan-Meier method, and statistical significance was determined by the log-rank test. $\mathrm{p}<0.05$ was considered statistically significant.

\section{Results \\ Clinical and Immunohistochemical Findings}

Of the 42 patients, 24 were male (54\%). The patients' median age at the time of surgery was 42 years (range $2-73$ years); 11 (26\%) of the patients were less than 15 years old. Five patients (12.0\%) underwent repeated operations. (The tumor specimens used for analysis, however, were all from the initial operation.) GTR was accomplished in 17 cases (40.5\%), subtotal in 19 (45.2\%), and partial in 6 (14.3\%). The duration of follow-up ranged from 4 to 170 months (median 46 months). Nineteen patients received adjuvant RT (fractionated radiotherapy was used in 16 patients and CyberKnife treatment in 3 patients). During the followup period, tumor recurrence was detected on MRI in 9 (21.4\%) patients.

As for radiological findings, the tumor volume ranged from 0.5 to $104.3 \mathrm{~cm}^{3}$ (median $9.5 \mathrm{~cm}^{3}$ ). Edema of the optic tract was observed in $9(90 \%)$ cases of papCP and in $13(40.6 \%)$ cases of adaCP. Calcification within the tumor was not observed in papCPs, but it was seen in 30 (93.8\%) cases of adaCP. High intensity of cyst fluid on T1-weighted MRI was observed in $1(10 \%)$ case of papCP and in 22 (68.8\%) cases of adaCP.

In regard to immunostaining, none of the cases of papCP showed positive immunohistochemical intranucle- 
TABLE 1. Clinical, radiological, and pathological data of the 42 patients included in the study

\begin{tabular}{|c|c|c|c|c|c|c|c|c|c|c|c|c|}
\hline $\begin{array}{l}\text { Case } \\
\text { No. }\end{array}$ & $\begin{array}{l}\text { Path } \\
\text { Findings }\end{array}$ & $\begin{array}{l}\text { Age (yrs), } \\
\text { Sex }\end{array}$ & Reop Case* & GTR & $\begin{array}{l}\text { Adj } \\
\text { RT }\end{array}$ & $\begin{array}{l}\mathrm{FU} \\
(\mathrm{mos})\end{array}$ & $\begin{array}{l}\text { Tumor Vol } \\
\text { in } \mathrm{cm}^{3} \dagger\end{array}$ & $\begin{array}{l}\text { Optic Tract } \\
\text { Edema }\end{array}$ & $\begin{array}{l}\text { T1WI Intensity } \\
\text { of Cyst Fluid }\end{array}$ & Calcification & $\begin{array}{c}\mathrm{IHC} \\
\beta \text {-Catenin }\end{array}$ & $\begin{array}{l}\text { IHC } \\
\text { VE1 }\end{array}$ \\
\hline Pap-1 & Pap & $32, \mathrm{~F}$ & - & - & + & 88 & 18.5 & + & Нypo & - & - & ++ \\
\hline Pap-2 & Pap & $56, M$ & - & - & + & 4 & 21.8 & + & Hyper & - & - & ++ \\
\hline Pap-3 & Pap & $54, \mathrm{M}$ & - & - & + & 6 & 12.5 & + & Hypo & - & - & ++ \\
\hline Pap-4 & Pap & $57, \mathrm{M}$ & - & - & - & 83 & 14.7 & + & Hypo & - & - & ++ \\
\hline Pap-5 & Pap & $64, \mathrm{~F}$ & - & - & + & 59 & 5.3 & + & Not cystic & - & - & ++ \\
\hline Pap-6 & Pap & $40, F$ & - & - & + & 16 & 9.8 & + & Not cystic & - & - & ++ \\
\hline Pap-7 & Pap & $20, F$ & - & + & - & 14 & 1.5 & + & Нypo & - & - & ++ \\
\hline Pap-8 & Pap & $43, F$ & - & + & - & 36 & 8.7 & + & Iso & - & - & ++ \\
\hline Pap-9 & Pap & $60, M$ & - & - & + & 8 & 3.9 & + & Hypo & - & - & ++ \\
\hline Pap-10 & Pap & $64, F$ & - & - & + & 29 & 1.3 & - & Iso & - & - & ++ \\
\hline Ada-1 & Ada & $71, \mathrm{~F}$ & - & - & + & 22 & 2.9 & - & Iso & - & + & - \\
\hline Ada-2 & Ada & $4, \mathrm{~F}$ & - & + & - & 20 & 7.0 & + & Нуро & + & ++ & - \\
\hline Ada-3 & Ada & $25, \mathrm{~F}$ & - & + & - & 33 & 18.9 & - & Hyper & + & ++ & - \\
\hline Ada-4 & Ada & $54, \mathrm{M}$ & - & + & - & 16 & 12.4 & + & Hyper & + & ++ & - \\
\hline Ada-5 & Ada & $7, M$ & - & - & - & 48 & 5.1 & + & Hypo & + & + & - \\
\hline Ada-6 & Ada & $20, M$ & - & - & + & 170 & 28.1 & - & Нypo & + & + & - \\
\hline Ada-7 & Ada & $49, \mathrm{~F}$ & - & - & - & 4 & 15.4 & - & Hyper & + & + & - \\
\hline Ada-8 & Ada & $59, \mathrm{M}$ & - & - & - & 56 & 23.2 & - & Hyper & + & ++ & - \\
\hline Ada-9 & Ada & $2, M$ & - & + & - & 21 & 104.3 & - & Iso & + & ++ & - \\
\hline Ada-10 & Ada & $5, F$ & - & + & - & 34 & 3.1 & - & Hyper & + & - & - \\
\hline Ada-11 & Ada & $3, M$ & - & - & - & 29 & 15.5 & - & Hyper & + & ++ & + \\
\hline Ada-12 & Ada & $62, F$ & + & - & + & 52 & 12.5 & + & Hyper & + & + & ++ \\
\hline Ada-13 & Ada & $56, \mathrm{M}$ & - & - & + & 110 & 5.8 & + & Нyро & + & + & - \\
\hline Ada-14 & Ada & $35, M$ & - & - & + & 95 & 12.9 & + & Hyper & + & + & - \\
\hline Ada-15 & Ada & $10, M$ & - & - & + & 66 & 5.3 & + & Hyper & + & + & + \\
\hline Ada-16 & Ada & $52, \mathrm{~F}$ & + & - & + & 65 & 19.4 & + & Hyper & + & - & - \\
\hline Ada-17 & Ada & $12, \mathrm{~F}$ & - & + & - & 64 & 10.2 & - & Hyper & + & - & ++ \\
\hline Ada-18 & Ada & $70, F$ & - & + & - & 33 & 2.5 & + & Iso & + & + & ++ \\
\hline Ada-19 & Ada & $59, \mathrm{M}$ & + & - & + & 69 & 26.9 & - & Hyper & + & ++ & ++ \\
\hline Ada-20 & Ada & $10, M$ & - & + & - & 47 & 16.7 & + & Hyper & + & ++ & - \\
\hline Ada-21 & Ada & $54, \mathrm{~F}$ & - & + & - & 57 & 2.0 & + & Нypo & + & - & - \\
\hline Ada-22 & Ada & $22, \mathrm{M}$ & + & + & - & 18 & 0.5 & - & Hyper & + & + & - \\
\hline Ada-23 & Ada & $64, \mathrm{M}$ & - & - & - & 30 & 6.9 & + & Hyper & + & + & - \\
\hline Ada-24 & Ada & $59, \mathrm{M}$ & + & + & - & 24 & 1.3 & - & Hyper & + & + & - \\
\hline Ada-25 & Ada & $7, M$ & - & + & - & 17 & 1.8 & - & Hyper & + & - & ++ \\
\hline Ada-26 & Ada & $16, M$ & - & + & - & 45 & 3.0 & - & Hypo & + & ++ & - \\
\hline Ada-27 & Ada & $73, \mathrm{~F}$ & - & + & - & 71 & 9.2 & - & Hyper & + & - & - \\
\hline Ada-28 & Ada & $25, M$ & - & - & - & 62 & 1.8 & - & Hyper & + & - & - \\
\hline Ada-29 & Ada & $15, M$ & - & - & + & 90 & 4.0 & - & Hyper & + & + & ++ \\
\hline Ada-30 & Ada & $44, \mathrm{M}$ & - & - & + & 93 & 39.0 & + & Iso & + & + & ++ \\
\hline Ada-31 & Ada & $10, M$ & - & - & + & 90 & 67.7 & - & Hyper & + & + & - \\
\hline Ada-32 & Ada & $6, F$ & - & - & + & 109 & 19.8 & - & Hyper & - & + & - \\
\hline
\end{tabular}

Ada = adamantinomatous; adj = adjunctive; FU = follow-up; hyper = hyperintense; hypo = hypointense; IHC = immunohistochemistry; iso = isointense; pap = papillary; path = pathological; RT = radiation therapy; T1WI = T1-weighted imaging .

${ }^{*}$ Indicates a case in which repeated operation was required.

$\dagger(\mathrm{AP} \times \mathrm{LR} \times \mathrm{SI}) / 2$, where AP is the anteroposterior measurement, $\mathrm{LR}$ is the left-right measurement, and $\mathrm{SI}$ is the superior-inferior measurement.

ar staining of $\beta$-catenin while all cases had strong positive staining of VE1. Among the cases of adaCP, 6 (19\%) showed strong positive immunohistochemical staining of
VE1 and 7 (22\%) were negative for $\beta$-catenin. These data are presented in Table 1 and in panels A-D of the Supplementary Figure (available online). 
TABLE 2. The mutation analysis data of patients with craniopharyngioma

\begin{tabular}{|c|c|c|c|c|c|c|c|}
\hline $\begin{array}{l}\text { Case } \\
\text { No. }\end{array}$ & $\begin{array}{c}\text { BRAF V600E Mutation } \\
\text { (NGS) }\end{array}$ & $\begin{array}{c}\text { Allele } \\
\text { Frequency }\end{array}$ & $\begin{array}{c}\text { BRAF V600E Mutation } \\
\text { (Sanger) }\end{array}$ & $\begin{array}{l}\text { CTNNB1 Mutation } \\
\text { (NGS) }\end{array}$ & $\begin{array}{c}\text { Allele } \\
\text { Frequency }\end{array}$ & $\begin{array}{l}\text { CTNNB1 Mutation } \\
\text { (Sanger) }\end{array}$ & $\begin{array}{l}\text { Final Eval of } \\
\text { CTNNB1 Mutation }\end{array}$ \\
\hline Pap-1 & + & $46.7 \%$ & + & & & - & \\
\hline Pap-2 & + & $37.8 \%$ & + & & & - & \\
\hline Pap-3 & + & $34.8 \%$ & + & & & - & \\
\hline Pap-4 & + & $34.3 \%$ & + & & & - & \\
\hline Pap-5 & + & $33.1 \%$ & + & & & - & \\
\hline Pap-6 & + & $32.5 \%$ & + & & & - & \\
\hline Pap-7 & + & $32.1 \%$ & + & & & - & \\
\hline Pap-8 & + & $29.4 \%$ & + & & & - & \\
\hline Pap-9 & + & $19.1 \%$ & + & & & - & \\
\hline Pap-10 & + & $10.2 \%$ & + & & & - & \\
\hline Ada-1 & & & - & T41I (122C>T) & $46.3 \%$ & T41I (122C>T) & + \\
\hline Ada-2 & & & - & T41I $(122 \mathrm{C}>\mathrm{T})$ & $41.2 \%$ & $\mathrm{~T} 41 \mathrm{I}(122 \mathrm{C}>\mathrm{T})$ & + \\
\hline Ada-3 & & & - & T41I $(122 \mathrm{C}>\mathrm{T})$ & $41.1 \%$ & $\mathrm{~T} 41 \mathrm{I}(122 \mathrm{C}>\mathrm{T})$ & + \\
\hline Ada-4 & & & - & $\mathrm{T} 41 \mathrm{I}(122 \mathrm{C}>\mathrm{T})$ & $38.0 \%$ & $\mathrm{~T} 41 \mathrm{I}(122 \mathrm{C}>\mathrm{T})$ & + \\
\hline Ada-5 & & & - & D32G $(95 A>G)$ & $35.5 \%$ & D32G $(95 A>G)$ & + \\
\hline Ada-6 & & & - & T41A $(121 A>G)$ & $35.5 \%$ & $\operatorname{T41A}(121 \mathrm{~A}>\mathrm{G})$ & + \\
\hline Ada-7 & & & - & T41I (122C>T) & $29.9 \%$ & T41I (122C>T) & + \\
\hline Ada-8 & & & - & $\mathrm{T} 41 \mathrm{I}(122 \mathrm{C}>\mathrm{T})$ & $28.9 \%$ & T41I $(122 \mathrm{C}>\mathrm{T})$ & + \\
\hline Ada-9 & & & - & S33F $(98 \mathrm{C}>\mathrm{T})$ & $26.5 \%$ & S33F $(98 \mathrm{C}>\mathrm{T})$ & + \\
\hline Ada-10 & & & - & G34E (101G>A) & $23.3 \%$ & G34E $(101 G>A)$ & + \\
\hline Ada-11 & & & - & D32V (95A>T) & $20.2 \%$ & D32V $(95 \mathrm{~A}>\mathrm{T})$ & + \\
\hline Ada-12 & & & - & G34R (100G>A) & $17.2 \%$ & G34R (100G>A) & + \\
\hline Ada-13 & & & - & $\mathrm{D} 32 \mathrm{Y}(94 \mathrm{G}>\mathrm{T})$ & $15.5 \%$ & $\mathrm{D} 32 \mathrm{Y}(94 \mathrm{G}>\mathrm{T})$ & + \\
\hline Ada-14 & & & - & S33C $(98 \mathrm{C}>\mathrm{G})$ & $13.4 \%$ & S33C $(98 C>G)$ & + \\
\hline Ada-15 & & & - & S33F $(98 \mathrm{C}>\mathrm{T})$ & $13.1 \%$ & S33F $(98 \mathrm{C}>\mathrm{T})$ & + \\
\hline Ada-16 & & & - & S33P $(97 T>C)$ & $12.2 \%$ & S33P $(97 T>C)$ & + \\
\hline Ada-17 & & & - & S33C $(98 C>G)$ & $10.9 \%$ & S33C $(98 \mathrm{C}>\mathrm{G})$ & + \\
\hline Ada-18 & & & - & S37C $(110 C>G)$ & $8.4 \%$ & S37C $(110 C>G)$ & + \\
\hline Ada-19 & & & - & D32N (94G>A) & $6.2 \%$ & D32N $(94 G>A)$ & + \\
\hline Ada-20 & & & - & D32N $(94 G>A)$ & $5.2 \%$ & D32N $(94 G>A)$ & + \\
\hline Ada-21 & & & - & S37F $(110 \mathrm{C}>\mathrm{T})$ & $4.8 \%$ & S37F (110C>T) & + \\
\hline Ada-22 & & & - & S33F $(98 \mathrm{C}>\mathrm{T})$ & $9.4 \%$ & - & - \\
\hline Ada-23 & & & - & S33F $(98 \mathrm{C}>\mathrm{T})$ & $6.5 \%$ & - & - \\
\hline Ada-24 & & & - & G34R $(100 G>C)$ & $3 \%$ & - & - \\
\hline Ada-25 & & & - & D32N $(94 G>A)$ & $2.8 \%$ & - & - \\
\hline Ada-26 & & & - & - & $0 \%$ & - & - \\
\hline Ada-27 & & & - & - & $0 \%$ & - & - \\
\hline Ada-28 & & & - & - & $0 \%$ & - & - \\
\hline Ada-29 & & & - & - & $0 \%$ & - & - \\
\hline Ada-30 & & & - & - & $0 \%$ & - & - \\
\hline Ada-31 & & & - & - & $0 \%$ & - & - \\
\hline Ada-32 & & & - & NA & NA & NA & NA \\
\hline
\end{tabular}

Eval = evaluation; $\mathrm{NA}=$ not available.

\section{BRAF and CTNNB1 Mutations in CP}

Next-generation sequencing (NGS) and validation by Sanger sequencing were used to detect $B R A F$ V600E and $C T N N B 1$ exon 3 mutations in DNA extracted from tumor tissue samples. These mutation analysis data are summarized in Table 2. The BRAF V600E mutation was detected in all $10(100 \%)$ cases of papCP, and CTNNB1 exon 3 mutations were detected in 21 of $31(68 \%)$ cases of adaCP; the sequence data of 1 case (ada-32) could not be analyzed. Among the detected CTNNB1 mutations, the 
TABLE 3. Summary of characteristics of adamantinomatous CPs according to CTNNB1 genetic status

\begin{tabular}{|c|c|c|c|}
\hline \multirow[b]{2}{*}{ Characteristic } & \multicolumn{2}{|c|}{ CTNNB1 Mutation Status } & \multirow{2}{*}{$\begin{array}{c}p \\
\text { Value }\end{array}$} \\
\hline & Positive & Negative & \\
\hline No. of cases & 21 & 10 & \\
\hline \multicolumn{4}{|l|}{ Clinical } \\
\hline Allele frequency (\%), mean & $22.5 \pm 13.0$ & $2.2 \pm 3.2$ & $<0.001$ \\
\hline \multicolumn{4}{|l|}{ Age } \\
\hline Median & 35 & 23.5 & 0.755 \\
\hline Range & $2-71$ & $7-73$ & \\
\hline Sex & & & 0.055 \\
\hline Male & 11 & 9 & \\
\hline Female & 10 & 1 & \\
\hline Tumor vol in $\mathrm{cm}^{3}$, mean & $16.7 \pm 21.1$ & $13.5 \pm 20.2$ & 0.072 \\
\hline GTR & $9(43 \%)$ & $5(50 \%)$ & 1.000 \\
\hline Adj RT & $8(38 \%)$ & $3(30 \%)$ & 0.712 \\
\hline Recurrence & $7(33 \%)$ & $1(10 \%)$ & 0.141 \\
\hline \multicolumn{4}{|l|}{ Radiological } \\
\hline Optic tract edema & $11(52 \%)$ & $220 \%)$ & 0.129 \\
\hline T1WI high intensity of cyst fluid & $13(62 \%)$ & $8(80 \%)$ & 0.429 \\
\hline Calcification & $20(95 \%)$ & $9(90 \%)$ & 1.000 \\
\hline \multicolumn{4}{|l|}{$\mathrm{IHC}$} \\
\hline$\beta$-catenin $++\&+$ & $17(81 \%)$ & $7(70 \%)$ & 0.652 \\
\hline Axin2 ++ \& + & $19(90 \%)$ & $7(70 \%)$ & 0.296 \\
\hline BMP4 ++ \& + & $16(76 \%)$ & $8(80 \%)$ & 1.000 \\
\hline MIB-1 (\%)* & $7.0 \pm 4.1$ & $7.1 \pm 3.9$ & 0.950 \\
\hline \multicolumn{4}{|l|}{ RT-PCR } \\
\hline $\begin{array}{l}\text { Axin2 (relative mRNA expres- } \\
\text { sion level), mean }\end{array}$ & $90.7 \pm 58.3$ & $49.0 \pm 42.6$ & 0.043 \\
\hline $\begin{array}{l}\text { BMP4 (relative mRNA expres- } \\
\text { sion level), mean }\end{array}$ & $2.1 \pm 1.6$ & $1.5 \pm 1.2$ & 0.186 \\
\hline
\end{tabular}

Values are numbers of cases (\%) unless otherwise indicated. Means are presented with SDs.

most frequent point mutation was T41I $(122 \mathrm{C}>\mathrm{T})$, which was seen in 6 of $31(19 \%)$ cases. Four cases were excluded from the CTNNB1 mutation-positive group after validation. The relationship between mutations and allele frequency was examined. Four of $8(50 \%)$ cases with NGS allele frequency of less than $10 \%$ were confirmed to be mutation positive by Sanger sequencing. All cases (17 of $17[100 \%]$ ) that had an allele frequency of more than $10 \%$ were confirmed to be mutation positive. Allele frequency of CTNNB1 mutation was significantly higher in mutationpositive cases $(22.5 \% \pm 13.0 \%)$ than in mutation-negative cases $(2.2 \% \pm 3.2 \%)(\mathrm{p}<0.001)$.

\section{Comparison of Cases of adaCP With and Without CTNNB1 Mutation}

\section{Clinical and Immunohistochemical Differences}

Correlations between CTNNB1 mutation positivity and clinical characteristics and immunohistochemical findings of adaCPs are summarized in Tables 1 and 3. No significant correlation was seen between CTNNBI mutation positivity and clinical and radiological factors (age, sex, tumor volume, GTR, optic tract edema, calcification, and high intensity of cyst fluid on T1-weighted MRI). The immunohistochemical findings of positive catenin staining and MIB-1 index did not correlate with CTNNB1 mutation positivity (Table 3 ). For analysis of PFS as a prognostic factor, we performed Kaplan-Meier survival analysis to assess the relationship between CTNNB1 mutation positivity and PFS in all of the cases except for the one in which the sequence data could not be analyzed (ada-32) and one with short follow-up (ada-7). The PFS was not significantly shorter in the mutation-positive group $(n=20 ; 1$-year PFS, 95\%; 2-year PFS, 68.9\%; 5-year PFS, 61.3\%) than in the mutation-negative group ( $\mathrm{n}=10$; 1-year PFS, 100\%; 2-year PFS, 100\%; 5-year PFS, 83.3\%) (log-rank test, $\mathrm{p}=0.141$ ) (Fig. 1A). We performed Kaplan-Meier survival analysis to assess the relationship between adjuvant RT and PFS (log-rank test, $\mathrm{p}=0.004$ ) (Fig. 1B).

Finally, we performed Kaplan-Meier survival analysis to assess the relationship between CTNNB1 mutation positivity and prognosis, excluding the 11 patients who had undergone adjuvant RT. The PFS was significantly shorter in the mutation-positive group $(\mathrm{n}=12$; 1-year PFS, 91.7\%; 2-year PFS, 45.7\%; 5-year PFS, 22.9\%) than in the mutation-negative group ( $\mathrm{n}=7$; 1-year PFS, 100\%; 2-year PFS, 100\%; 5-year PFS, 66.7\%) (log-rank test, $\mathrm{p}=0.031$ ) (Fig. 1C). There was no significant association between $C T N$ NB1 mutation and other clinical characteristics, including age, sex, and extent of resection.

\section{Differences of Wht Signal Expression}

In order to examine the biological effect of the $C T N$ NB1 mutation on Wnt signaling, mRNA expression of Axin2 and BMP4 was evaluated. AdaCPs with or without a CTNNB1 mutation seemed to have higher mRNA expression levels of Axin2 and BMP4 compared with papCPs. The mRNA expression level of Axin2 of adaCPs with CTNNB1 mutations was significantly higher than that of adaCPs without mutations ( $\mathrm{p}<0.05$, Fig. 2 left). There was no association between adaCPs with CTNNBI mutations and $B M P 4$ mRNA expression ( $\mathrm{p}=0.186$, Fig. 2 right). The statistical data are shown in Fig. 2. The immunohistochemical findings of Axin2 and BMP4 had no significant correlation with CTNNBI mutation positivity (Table 3) (Supplementary Figure panels E and F).

\section{Discussion}

In this study, we examined $B R A F$ and $C T N N B 1$ mutations in 42 CPs using 2 different sequence analyses and investigated the association between CTNNB1 mutation and the clinical, pathological, radiological, and biological characteristics of CPs, as well as its relationship to the prognosis of these patients. We found that there was a significant correlation between the presence of CTNNBI mutation and the mRNA expression level of Axin2 in adaCPs. We also found that patients with adaCPs harboring a CTNNB1 mutation who did not undergo adjuvant RT had shorter PFS. These results suggest that presence of a $C T N N B 1$ mutation affects $\mathrm{Wnt} / \beta$-catenin signaling pathway activation and is a risk factor for poor PFS in patients with adaCP. 

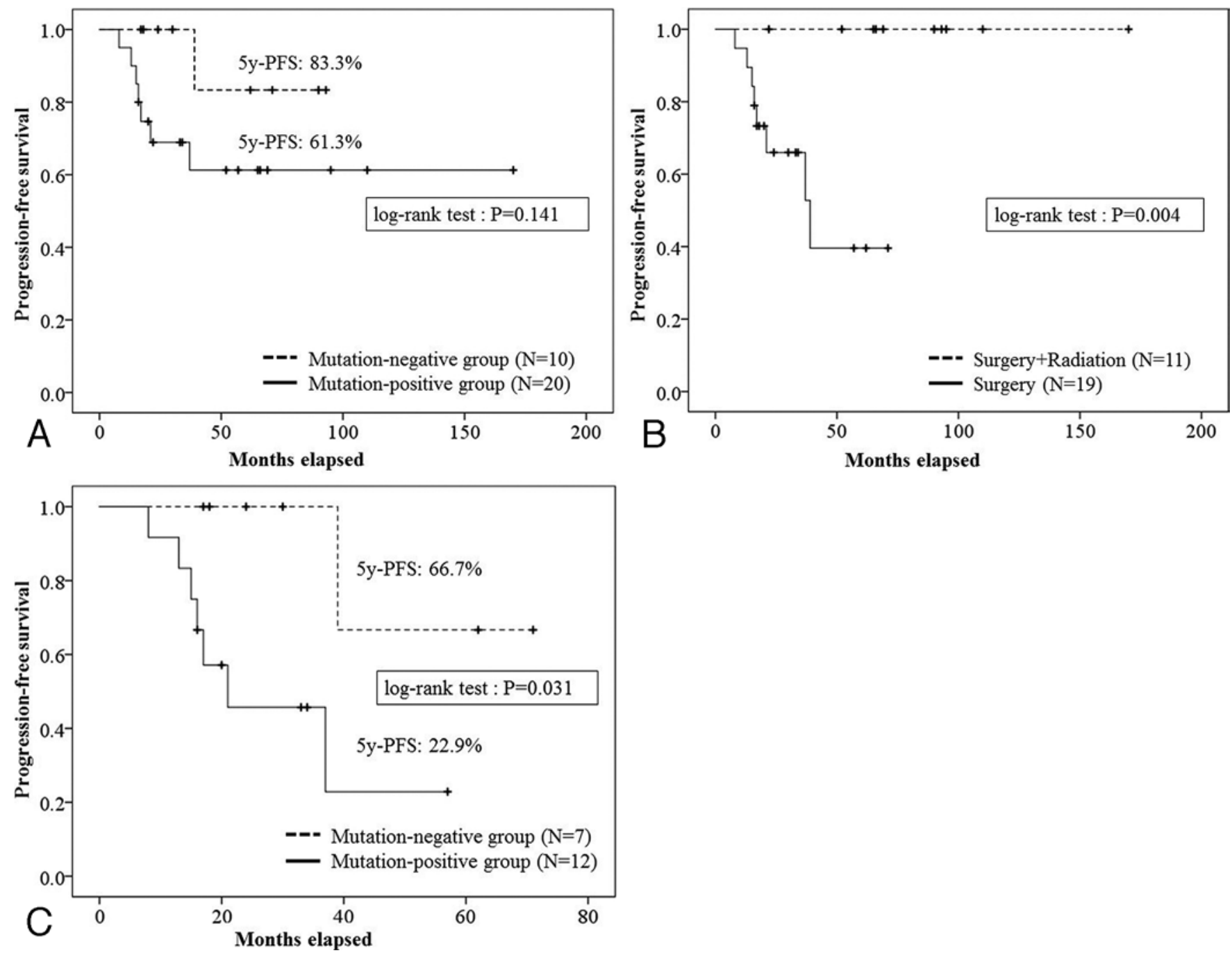

FIG. 1. A: Correlation of CTNNB1 mutation positivity with PFS in all adamantinomatous craniopharyngioma (adaCP) cases. B: Correlation of PFS with surgery or surgery plus adjuvant RT in patients with adaCP. C: Correlation of CTNNB1 mutation positivity with PFS in patients with adaCP, excluding patients treated with adjuvant RT. PFS was assessed using the Kaplan-Meier method, and statistical significance was determined by the log-rank test.

Many studies have suggested the important role played by $\beta$-catenin in tumorigenesis, progression, and invasion of adaCP. $2,11,13,24$ Various analyses revealed that genetic mutation in exon 3 of the $\beta$-catenin gene CTNNBI is present in $64 \%-99 \%$ of patients with adaCPs..$^{1,3,4,12}$ However, the existence of adaCP without CTNNBI mutation had not previously been confirmed and the clinical significance of CTNNB1 mutation in adaCP patients is still unclear. In the present study, we validated the sequencing results using 2 different methods. Compared with an NGS mutation allele frequency of more than 10\%, judgment of Sanger sequencing was more difficult if the NGS mutation allele frequency was less than $10 \%$ because of the lower peak. Finally, we confirmed the existence of adaCP without CTNNB1 mutation and the frequency of the mutation was approximately $70 \%$.

The CTNNB1 mutation affects the functional domains of $\beta$-catenin that interact with its regulators, including glycogen synthase kinase 3- $\beta$ (GSK3- $\beta$ ). These sites encode a degradation targeting motif, the mutation of which confers resistance to the $\beta$-catenin destruction complex. ${ }^{3,26}$ As a result, the $\beta$-catenin protein accumulates and translocates to the nucleus, where it facilitates transcription of $\beta$-catenin target genes and Wnt-regulated cellular processes. ${ }^{2-4,16}$ Furthermore, it was shown that EGFR and SHH signaling pathways are also upregulated in adaCP and are associated with tumor cell migration. ${ }^{9,10}$ Although many reports have highlighted these $\beta$-catenin mechanisms in adaCP, there are only a few studies that have investigated clinical and radiological differences between CTNNB1 mutation-positive and -negative adaCPs. ${ }^{9}$ We found that the CTNNBI mutation of adaCP affects 2 things: disease behavior as seen biologically and clinically, such as mRNA expression level of Axin2, and PFS. Analysis of the relationship of CTNNB1 mutation and PFS showed that adjuvant RT had the most significant effect on PFS in our study. We explain the reason why the difference of PFS was observed only after excluding the group that received RT as follows. 

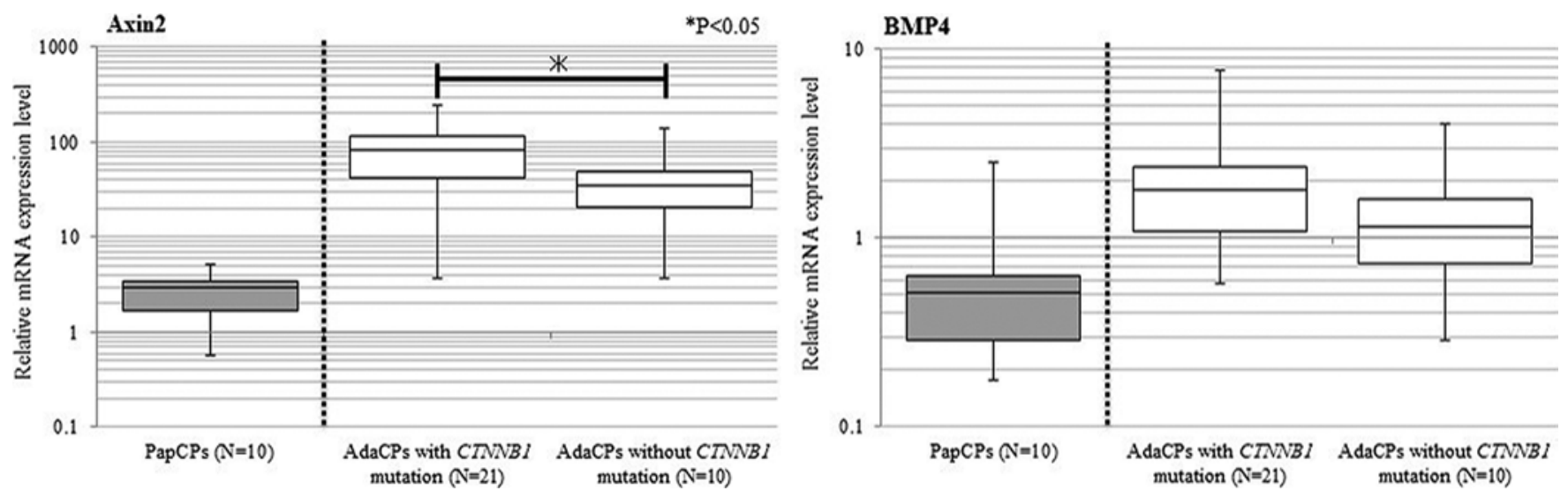

FIG. 2. Relative mRNA expression in papillary and adamantinomatous CPs (papCPs and adaCPs, respectively) with CTNNB1 mutations and without mutations. Left: The adaCPs with CTNNB1 mutations had higher mRNA expression levels of Axin2 compared with adaCPs without mutations $(p=0.043)$. Right: There was no association between disease-free survival and BMP4 mRNA expression $(p=0.186)$. These data were evaluated by the Mann-Whitney U-test and presented as median (horizontal line), interquartile range (box), 5th-95th percentile (whiskers); ${ }^{*} p<0.05$.

CTNNB1 mutation was associated with a higher rate of tumor recurrence in patients who did not receive RT in our study. However, RT strongly prevented recurrence whether the tumor was CTNNBI mutation positive or not. Accordingly, the difference of PFS was not observed between the entire groups of patients with and without CTNNB1 mutation because of the decreased rate of recurrence due to RT in the group with $C T N N B 1$ mutation.

Axin2 is a recognized inhibitor and target gene of $\beta$ catenin..$^{14}$ BMP4 expression is enhanced in tumors with oncogenic $\beta$-catenin elevation. ${ }^{15}$ The Axin 2 and the BMP4 proteins are assumed to be markers of $\mathrm{Wnt} / \beta$-catenin signaling pathway activation. ${ }^{11}$ However, it was uncertain whether mRNA expression levels of the 2 genes are elevated. We chose these 2 genes and their corresponding proteins as an indicator of $\mathrm{Wnt} / \beta$-catenin signaling pathway. First, we confirmed that these 2 genes were upregulated in adaCPs compared with papCPs, as described previously. ${ }^{11}$ Next, we found that among adaCPs, Axin 2 mRNA expression but not BMP4 mRNA expression was significantly higher in the CTNNBI mutation-positive group compared with the $C T N N B 1$ mutation-negative group.

Elevation of the mRNA level of Axin2 indicates an activated negative feedback loop that opposes Wnt/ $\beta$ catenin signaling in adaCP. Hölsken et al. reported that $B M P 4$ mRNA expression of adaCPs was higher than that of papCPs, but the difference of BMP4 mRNA expression between papCPs and adaCPs was significantly lower than that of Axin $2 .{ }^{11}$ In our study, we confirmed that BMP4 mRNA expression of adaCPs was significantly higher than that of papCPs. However, there was no difference in $B M P 4$ mRNA expression between adaCPs with a CTNNB1 mutation and those without. We assumed that a small sample number is the reason why the expected result-that $B M P 4$ mRNA expression of adaCPs with a CTNNB1 mutation is significantly higher than that of those without-was not obtained. The discrepancy between the statistical analysis of protein and mRNA expression in Axin2 depends on the method of evaluation used for these analyses and the sensitivity and specificity of the experiment. In evaluating the IHC results, digitization and comparing the degree of the staining was very difficult and not sensitive. It was presumably difficult to discover a slight difference in protein expression between adaCPs with CTNNBI mutation and those without the mutation by IHC only. As for detection of Axin 2 expression, mRNA level by RT-PCR had a superior sensitivity compared with examination of protein level by IHC. To reanalyze and evaluate IHC sensitivity, we applied another monoclonal Axin2 antibody (ab109307, dilution 1:150; Abcam) to detect Axin2 protein in tissue. In the reanalysis of Axin2 protein semiquantitative expression, there was no significant difference between adaCPs with CTNNB1 mutation and those without CTNNB1 mutation (data not shown). Finally, we supposed that the difference of specificity and sensitivity in each assay was the cause of the discrepancy.

Many clinical, pathological, radiological, and biological factors predictive of CP recurrence have been suggested, ${ }^{25}$ such as male sex, occurrence in childhood,$^{20}$ incomplete removal, ${ }^{28}$ no RT, ${ }^{22}$ larger tumor size, ${ }^{7}$ presence of whorllike arrays ${ }^{28}$ high expression of $\mathrm{p} 53,{ }^{28}$ and higher levels of $S M O$ mRNA expression. ${ }^{9}$ Moreover, $\mathrm{Li}$ et al. reported that aberrant $\beta$-catenin expression significantly correlated with a poor survival rate in patients with CP and CTNNBI mutation. ${ }^{17}$ However, their study included both adaCPs and papCPs; therefore, the biological marker related to PFS in adaCPs remains unclear. In our study, the PFS was shorter in the CTNNB1 mutation-positive group than in the mutation-negative group of adaCPs that had not been subjected to radiotherapy. We consider that $C T N N B 1$ mutation, which correlates with aberrant $\beta$-catenin expression, is somehow related to prognosis and recurrence of adaCPs.

We showed that CTNNBI mutation leads to activation of the Wnt/ $\beta$-catenin signaling by comparing the mRNA expression level of Axin2 of CTNNB1 mutation-positive and -negative samples. Many previous reports described that $\mathrm{Wnt} / \beta$-catenin signaling activation plays an important role in the pathogenesis of adaCPs and tumor invasion..$^{9,11,17}$ These studies suggest that $\mathrm{Wnt} / \beta$-catenin and SHH signaling activation induced subsequent gene activation, includ- 
ing activation of Axin2, BMP4, SMO, and GLII. However, the detailed mechanism after nuclear accumulation of $\beta$-catenin has not been elucidated due to the interaction of these molecules, and we need to further investigate the function and mechanism of these molecules.

Axin2 plays an important role in the regulation of the stability of $\beta$-catenin in the Wnt/ $\beta$-catenin signaling pathway and mutations of Axin2 have been associated with colorectal cancer. ${ }^{14}$ This gene is recognized as a negative regulator of the $\mathrm{Wnt} / \beta$-catenin signaling pathway. ${ }^{14}$ It is unclear whether Axin2 has the potential to become a direct target of molecularly targeted drugs. There is a report of inhibition of brain tumor growth by downregulation of the Wnt $/ \beta$-catenin signaling pathway by microRNA. ${ }^{29}$ Further investigations targeting the $\mathrm{Wnt} / \beta$-catenin signaling pathway, including such possible targets as Axin2, are needed to improve the prognosis of patients with adaCPs.

Our study has limitations. The number of patients was small and the treatment strategy was heterogeneous. The first choice of surgical approach changed from transcranial surgery to endoscopic endonasal surgery during the study period. Moreover, we recently have tended to choose endoscopic endonasal surgery for the treatment of recurrent tumors, considering the reported excellent results of endoscopic endonasal surgery for recurrent CPs. ${ }^{6}$ Furthermore, we could not completely exclude the possibility of selection bias for RT. Regarding technical aspects of the study, in patients with adaCPs who underwent partial resection, the quality and quantity of tissue sample were not sufficient. On the other hand, we tended to collect more optimal tissue samples in a recent study period. Because this was a retrospective single-center study, institutionspecific factors may limit generalizability; a prospective multicenter study is needed.

\section{Conclusions}

This study demonstrated biological and clinical differences between adaCPs with a CTNNBI mutation and those without. The mRNA expression level of Axin2 was higher in the CTNNB1 mutation-positive group than in the mutation-negative group. Although adjuvant RT had the most significant effect on PFS, in patients with adaCP, except for those who received adjuvant RT, PFS was shorter in the CTNNB1 mutation-positive group than in the mutation-negative group. These results raise the possibility that the CTNNB1 mutation may be associated with disease recurrence and genes related to the Wnt $/ \beta$-catenin signaling pathway. The CTNNB1 mutation is associated with adaCP and can be a target for treatment of these tumors.

\section{Acknowledgments}

We thank Yoshiko Tsukada, Makiko Miyakawa, and Kyoko Agemura for their excellent technical assistance.

\section{References}

1. Brastianos PK, Taylor-Weiner A, Manley PE, Jones RT, DiasSantagata D, Thorner AR, et al: Exome sequencing identifies BRAF mutations in papillary craniopharyngiomas. Nat Genet 46:161-165, 2014

2. Buslei R, Hölsken A, Hofmann B, Kreutzer J, Siebzehnrubl F,
Hans V, et al: Nuclear beta-catenin accumulation associates with epithelial morphogenesis in craniopharyngiomas. Acta Neuropathol 113:585-590, 2007

3. Buslei R, Nolde M, Hofmann B, Meissner S, Eyupoglu IY, Siebzehnrübl F, et al: Common mutations of beta-catenin in adamantinomatous craniopharyngiomas but not in other tumours originating from the sellar region. Acta Neuropathol 109:589-597, 2005

4. Cani CMG, Matushita H, Carvalho LRS, Soares IC, Brito LP, Almeida MQ, et al: PROP1 and CTNNB1 expression in adamantinomatous craniopharyngiomas with or without $\beta$ catenin mutations. Clinics (São Paulo) 66:1849-1854, 2011

5. Committee of the Brain Tumor Registry of Japan: Brain Tumor Registry of Japan: (2005-2008) 14th edition. Neurol Med Chir (Tokyo) 57 (Suppl 1):9-102, 2017

6. Dhandapani S, Singh H, Negm HM, Cohen S, Souweidane MM, Greenfield JP, et al: Endonasal endoscopic reoperation for residual or recurrent craniopharyngiomas. J Neurosurg 126:418-430, 2017

7. Fahlbusch R, Honegger J, Paulus W, Huk W, Buchfelder M: Surgical treatment of craniopharyngiomas: experience with 168 patients. J Neurosurg 90:237-250, 1999

8. Fernandez-Miranda JC, Gardner PA, Snyderman CH, Devaney KO, Strojan P, Suárez C, et al: Craniopharyngioma: a pathologic, clinical, and surgical review. Head Neck 34:1036-1044, 2012

9. Gomes DC, Jamra SA, Leal LF, Colli LM, Campanini ML, Oliveira RS, et al: Sonic Hedgehog pathway is upregulated in adamantinomatous craniopharyngiomas. Eur J Endocrinol 172:603-608, 2015

10. Hölsken A, Gebhardt M, Buchfelder M, Fahlbusch R, Blümcke I, Buslei R: EGFR signaling regulates tumor cell migration in craniopharyngiomas. Clin Cancer Res 17:4367-4377, 2011

11. Hölsken A, Kreutzer J, Hofmann BM, Hans V, Oppel F, Buchfelder M, et al: Target gene activation of the Wnt signaling pathway in nuclear beta-catenin accumulating cells of adamantinomatous craniopharyngiomas. Brain Pathol 19:357-364, 2009

12. Hölsken A, Sill M, Merkle J, Schweizer L, Buchfelder M, Flitsch J, et al: Adamantinomatous and papillary craniopharyngiomas are characterized by distinct epigenomic as well as mutational and transcriptomic profiles. Acta Neuropathol Commun 4:20, 2016

13. Hussain I, Eloy JA, Carmel PW, Liu JK: Molecular oncogenesis of craniopharyngioma: current and future strategies for the development of targeted therapies. J Neurosurg 119:106-112, 2013

14. Jho EH, Zhang T, Domon C, Joo CK, Freund JN, Costantini $\mathrm{F}$ : Wnt/beta-catenin/Tcf signaling induces the transcription of Axin2, a negative regulator of the signaling pathway. Mol Cell Biol 22:1172-1183, 2002

15. Kim JS, Crooks H, Dracheva T, Nishanian TG, Singh B, Jen $\mathrm{J}$, et al: Oncogenic beta-catenin is required for bone morphogenetic protein 4 expression in human cancer cells. Cancer Res 62:2744-2748, 2002

16. Larkin SJ, Ansorge O: Pathology and pathogenesis of craniopharyngiomas. Pituitary 16:9-17, 2013

17. Li Z, Xu J, Huang S, You C: Aberrant membranous expression of $\beta$-catenin predicts poor prognosis in patients with craniopharyngioma. Ann Diagn Pathol 19:403-408, 2015

18. Louis DN, Perry A, Reifenberger G, von Deimling A, Figarella-Branger D, Cavenee WK, et al: The 2016 World Health Organization Classification of Tumors of the Central Nervous System: a summary. Acta Neuropathol 131:803-820, 2016

19. Marucci G, de Biase D, Zoli M, Faustini-Fustini M, Bacci A, Pasquini E, et al: Targeted BRAF and CTNNB1 next-generation sequencing allows proper classification of nonadenomatous lesions of the sellar region in samples with limiting 
amounts of lesional cells. Pituitary 18:905-911, 2015

20. Minamida Y, Mikami T, Hashi K, Houkin K: Surgical management of the recurrence and regrowth of craniopharyngiomas. J Neurosurg 103:224-232, 2005

21. Mortini P, Gagliardi F, Boari N, Losa M: Surgical strategies and modern therapeutic options in the treatment of craniopharyngiomas. Crit Rev Oncol Hematol 88:514-529, 2013

22. Nagahata M, Hosoya T, Kayama T, Yamaguchi Kl: Edema along the optic tract: a useful MR finding for the diagnosis of craniopharyngiomas. AJNR Am J Neuroradiol 19:17531757,1998

23. Nagahata M, Hosoya T, Kayama T, Yamaguchi K: Edema along the optic tract: a useful MR finding for the diagnosis of craniopharyngiomas. Am J Neuroradiol 19:1753-1757, 1998

24. Oikonomou E, Barreto DC, Soares B, De Marco L, Buchfelder M, Adams EF: Beta-catenin mutations in craniopharyngiomas and pituitary adenomas. J Neurooncol 73:205209, 2005

25. Prieto R, Pascual JM, Subhi-Issa I, Jorquera M, Yus M, Martínez R: Predictive factors for craniopharyngioma recurrence: a systematic review and illustrative case report of a rapid recurrence. World Neurosurg 79:733-749, 2013

26. Robinson LC, Santagata S, Hankinson TC: Potential evolution of neurosurgical treatment paradigms for craniopharyngioma based on genomic and transcriptomic characteristics. Neurosurg Focus 41(6):E3, 2016

27. Rosemberg S, Fujiwara D: Epidemiology of pediatric tumors of the nervous system according to the WHO 2000 classification: a report of 1,195 cases from a single institution. Childs Nerv Syst 21:940-944, 2005

28. Tena-Suck ML, Salinas-Lara C, Arce-Arellano RI, RembaoBojórquez D, Morales-Espinosa D, Sotelo J, et al: Clinicopathological and immunohistochemical characteristics associated to recurrence/regrowth of craniopharyngiomas. Clin Neurol Neurosurg 108:661-669, 2006
29. Zhang W, Shen C, Li C, Yang G, Liu H, Chen X, et al: miR577 inhibits glioblastoma tumor growth via the Wnt signaling pathway. Mol Carcinog 55:575-585, 2016

\section{Disclosures}

This work was supported in part by a Grant-in-Aid for Scientific Research (to S. Takano, No. 15H04947) from the Ministry of Education, Culture, Sports, Science, and Technology of Japan.

\section{Author Contributions}

Conception and design: Hara. Acquisition of data: Takano, Hara, Akutsu, Kino, Tanaka, Miyamoto. Analysis and interpretation of data: Takano, Hara, Sakamoto, Hiyama, Masumoto. Drafting the article: Hara. Critically revising the article: Takano, Hara, Akutsu. Reviewed submitted version of manuscript: Takano, Hara, Akutsu. Approved the final version of the manuscript on behalf of all authors: Takano. Statistical analysis: Hara. Administrative/technical/material support: Hattori, Sakata-Yanagimoto, Chiba. Study supervision: Ishikawa, Matsumura.

\section{Supplemental Information}

Online-Only Content

Supplemental material is available with the online version of the article.

Supplementary Figure. https://thejns.org/doi/suppl/10.3171/ 2018.3.JNS172528.

\section{Correspondence}

Shingo Takano: University of Tsukuba, Ibaraki, Japan. shingo4@ md.tsukuba.ac.jp. 